\title{
A Randomised Interventional Study to Compare Autologous and Nonautologous Dural Substitutes Among Traumatic Brain Injury Patients
}

\author{
Vir Abhimanyu Pandit ${ }^{1}$ Rajesh Kumar Sharma² Suryanarayanan Bhaskar ${ }^{3}$ \\ Amanjeet Singh Kindra ${ }^{4}$ Ajay Choudhary ${ }^{2}$ LN Gupta ${ }^{2}$
}

1Department of Neurosurgery, Narayan Medical College and Hospital, Jamuhar, Rohtas, Bihar, India

2Department of Neurosurgery, Atal Bihari Vajpayee Institute of Medical Sciences (ABVIMS), Dr Ram Manohar Lohia Hospital, New Delhi, India

${ }^{3}$ Department of Neurosurgery, All Indian Institute of Medical Science, Jodhpur, India

${ }^{4}$ Shah Neuro and Trauma Centre Kaithal, Haryana, India
Address for correspondence Rajesh Kumar Sharma, MBBS, MS, MCh, Department of Neurosurgery, Atal Bihari Vajpayee Institute of Medical Sciences (ABVIMS), Dr Ram Manohar Lohia Hospital, New Delhi 110001, India (e-mail: drsrk22@gmail.com).

Indian J Neurotrauma:2021;18:26-31

\begin{abstract}
Keywords

- dural substitutes

- galea-pericranium

- traumatic brain injury

Objectives To determine and compare the effectiveness and safety of galeapericranium autologous dural graft with nonautologous polypropylene (G-patch) dural substitute among traumatic brain injury (TBI) patients.

Methods A prospective interventional randomized comparative study was conducted at the Department of Neurosurgery from November 2013 to March 2015 after obtaining approval from the institutional ethics committee. The study population included 50 cases of TBI which were divided into two groups of 25 each by the randomization technique and were treated either with autologous duraplasty (galea-pericranium) or nonautologous polypropylene (G-patch) dural substitute. The outcomes measured were time to duraplasty, blood loss, hospital stay, and the incidence of complications with the two techniques. The data were entered in a MS Excel spreadsheet and analysis was done using Statistical Package for Social Sciences (SPSS) version 21.0. A p value of $<0.05$ was considered statistically significant. Results The average time to harvest galea-pericranium was 5 minutes. Compared with the patients undergoing $\mathrm{G}$-patch, the patients in group pericranium had comparable duraplasty time (minutes) (34.32 vs. $27.80, p=0.44$ ), significantly lower drain output (54.8 vs. $74.5, p=0.017$ ), comparable blood loss (322 vs. $308, p=0.545$ ), comparable blood transfusion ( $24 \%$ vs. $16 \%, p=0.48$ ), significantly lesser duration of hospital stay ( 8.6 vs. $10.44, p=0.028)$, comparable wound infection ( $8 \%$ vs. $16 \%$, $p=0.384)$, and comparable cerebrospinal fluid (CSF) leak ( $0 \%$ vs. $8 \%, p=0.149)$.

Conclusion The study showed that galea-pericranium and polypropylene dural patch are equally effective and safe dural substitutes in providing a dural seal to minimize the CSF leaks and infections among posttraumatic brain injury patients.
\end{abstract}

\section{Introduction}

Traumatic brain injuries (TBIs) are complex injuries which occur at rate of 69 million/year (WHO data) and accounts for

published online

September 29, 2020
DOI https://doi.org/

10.1055/s-0040-1717216 ISSN 2277-954X.
5 million deaths every year. ${ }^{1}$ They are a significant cause of worldwide young deaths, of which $70 \%$ burden rests on the developing country as ours. ${ }^{2}$ A raised intracranial pressure (ICP) due to brain concussion and hemorrhage demands

(C)2020. Neurotrauma Society of India.

This is an open access article published by Thieme under the terms of the Creative Commons Attribution-NonDerivative-NonCommercial-License, permitting copying and reproduction so long as the original work is given appropriate credit. Contents may not be used for commercial purposes, or adapted, remixed, transformed or built upon. (https://creativecommons.org/licenses/by-nc-nd/4.0/).

Thieme Medical and Scientific Publishers Pvt. Ltd. A-12, 2nd Floor, Sector 2, Noida-201301 UP, India 
immediate attention which is initially managed conservatively (hyperosmolar agents, analgesia, deep sedation) with minimal invasiveness (ventriculostomy) or by more invasive decompressive craniectomy, creating dura mater defects. ${ }^{3}$ The neurosurgeons are expected to cover the gap in a watertight fashion, with the use of dural substitutes in what constitutes as duraplasty.

The use of a dural substitute (instead of leaving it uncovered) has been reinforced in the latest retrospective study in China (2018) which found that use of tight closure had significantly less subcutaneous hematoma $(4.01 \%$ vs. $13.02 \%$, $p=0.004)$; significantly less infection (5.64\% vs. $12.5 \%$, $p=0.021$ ), significantly less cerebrospinal fluid (CSF) leakage (5.13\% vs. $13.02 \%, p=0.012$ ), and significantly less seizures ( $3.08 \%$ vs. $10.42 \%, p=0.007$ ). The only problem with artificial dural substitute was the occurrence of increased foreign body reaction and high cost. ${ }^{3}$

An ideal dural substitute should be non-neurotoxic, nonimmunogenic, noninflammatory, nonviral/prion, nonadhesive to other tissues, watertight, viscoelastic, and biomechanically resistant. ${ }^{4}$ Human dura has collagen and elastin and is the most appropriate viscoelastic dural substitute, which fulfills all the criteria, as it is autologously derived from the galea-pericranium, fascia lata, fat, or temporal fascia. Few of the shortcomings in the use of autologous human dura include prolonged time of collection and retrieval, creation of an additional incision, and insufficiency to fill large dural defects. ${ }^{5}$ Despite that, other complications such as subcutaneous fluid collections, wound infections, CSF leak are found minimal among the autologous dural substitutes. ${ }^{5}$

In this fast world, where newer research and technology has been continuing, the need and the use of nonautologous dural substitutes are increasing, since it is easily available, feasible, less time consuming, avoids an additional patient incision, and is rapidly deployable. ${ }^{5}$ The research has led to the introduction of allografts (human cadavers), xenografts comprising pericardium, peritoneum, dermis, and fascia lata from bovine, porcine, ovine, or equine donors; ${ }^{6}$ and synthetics with materials such as Dacron, reconstituted collagen foil, expanded polytetrafluoroethylene (ePTFE), and absorbable and nonabsorbable polymers. ${ }^{3}$

However, the ongoing research fails to address the superiority of xenografts over the autografts. The increasing development of newer synthetics has also increased the cost associated with duraplasty. In addition, studies comparing the autografts and xenografts are sparse among the patients with TBI. Thus, we conducted this comparative interventional study to determine and compare the effectiveness and safety of galea-pericranium dural graft with G-patch dural substitute during duraplasty among TBI patients.

\section{Methods}

A prospective interventional randomized comparative study was conducted at the Department of Neurosurgery from November 2013 to March 2015. Approval for conducting study was obtained from the institutional ethics committee and informed consent was received from all study participants.
The study population included 50 cases of TBIs that were admitted to the hospital on an emergency/elective basis and underwent duraplasty. The exclusion criteria included patients with age $<18$ and $>65$ years, those with compound depressed fracture, posterior fossa craniotomy, chronic medical disorders such as immunocompromised, uncontrolled diabetes mellitus, chronic liver disease, and chronic renal disease, and those who underwent redo surgeries. Supratentorial neoplasms, meningiomas, and cerebral aneurysms, in which duraplasty was necessary were not included.

The calculation of the sample size was based on the study of Sun et al, who observed that mean value of blood loss in artificial dura materials (ADM) group was $139.63 \pm 37.46 \mathrm{~mL} .^{3}$ Taking those values as reference and assuming difference in blood loss of $30 \mathrm{~mL}$ between the two groups, the minimum required sample size with $80 \%$ power of study and $5 \%$ level of significance was calculated out to be 25 patients in each study group. So, total sample size taken was 50 patients ( 25 patients per group).

Before the start of the study, 50 study patients were divided into two groups of 25 each with the help of the randomization technique and sealed envelope system which contained randomly generated treatment allocations slips. Once a patient consented to enter a trial, an envelope was opened, and the patient was then offered the allocated treatment regimen.

Study population included patients who underwent duraplasty with galea-pericranium (group pericranium) or nonautologous polypropylene (group G-patch) dural patch. The surgical procedure was performed by the same surgeon to standardize the technique.

Surgery was conducted on the basis of clinicoradiological diagnosis. CT head plain was done in all cases for diagnosis and surgical planning. Under general anesthesia, flap craniotomy was done, depressed fracture segment if any was elevated, and durotomy was done for hematoma evacuation. Duraplasty was planned after evacuation of intradural pathology. If brain was bulging even after complete evacuation of the intradural lesion like parenchymal hematoma and acute subdural hematoma, duraplasty was planned. Time used for galea-pericranium collection was measured in all procedures in which it was adopted by means of operating room timer. The dural substitute was sutured with the native dura with either interrupted nonabsorbable suture or continuous suture in a watertight manner $(-$ Fig. 1$)$. Wound was sutured in layers and dressing done under aseptic precaution. Postoperative daily wound examination was done. If patients developed any specific sign and symptoms of meningitis, lumbar puncture (LP) was done. If there was CSF leak from wound, local wound suturing with polypropylene, dressing, and CT was done to rule out hydrocephalus. If hydrocephalus was present, CSF diversion was done.

Postoperative antibiotics were given till all drains were removed. Suture removal was done on day 8 or later after wound assessment. After neurological stabilization, patients were discharged and followed-up for a minimum period of 30 days. Patients who survived $<1$ month were not included in the study. 
The outcomes measured were time to duraplasty, blood loss, hospital stay, and incidence of complications with the two techniques.

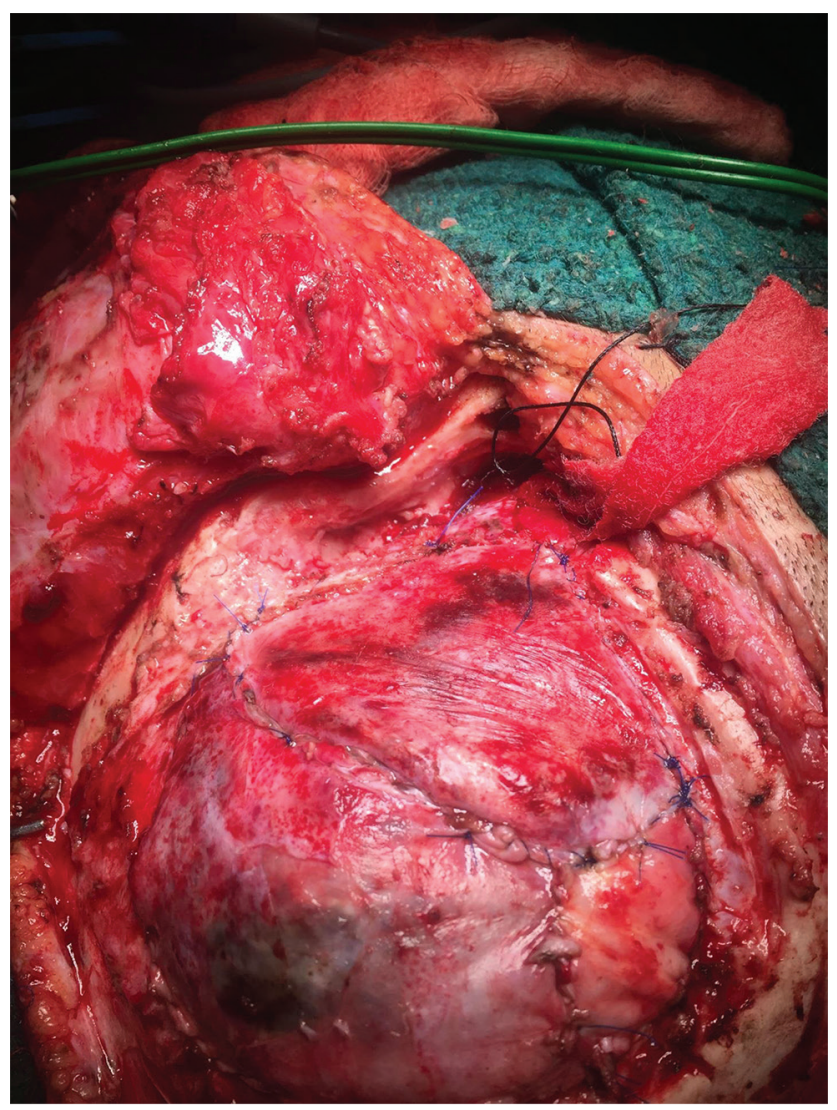

Fig. 1 A traumatic brain injury (TBI) case whose dural repair was done using autologous dural substitute.
Statistical analysis: Categorical variables were presented in number and percentage (\%) and continuous variables were presented as mean $\pm \mathrm{SD}$. Quantitative variables were compared using unpaired $t$-test between the two groups. Qualitative variables were compared using Chi-Square test/Fisher's exact test. A $p$ value of $<0.05$ were considered statistically significant. The data were entered in a MS Excel spreadsheet and analysis was done using Statistical Package for Social Sciences (SPSS) version 21.0.

\section{Results}

The sociodemographic parameters are shown in - Table $\mathbf{1}$. Compared with the patients undergoing G-patch, the patients who underwent pericranium autologous graft had comparable age $(p=0.484)$, comparable number of males ( $88 \%$ vs. $80 \%)$ and females ( $12 \%$ vs. $20 \%)(p=0.702$ for both), comparable number of patients with headache ( $60 \%$ vs. $32 \%, p=0.089$ ), vomiting ( $44 \%$ vs. $52 \%, p=0.777$ ), and loss of consciousness ( $40 \%$ vs. $68 \%, p=0.089$ ). However, there was a significant difference in the type of head injury among the two groups, as cases of minimal and severe head injuries were more in group pericranium but cases of mild and less moderate head injury were more in group G-patch $(p=0.036)$.

Among the operative parameters, the average time to harvest galea-pericranium was 5 minutes. Compared with the patients undergoing G-patch, the patients in group pericranium had comparable duraplasty time (minutes) (34.32 vs. $27.80, p=0.44$ ), significantly lower drain output ( 54.8 vs. $74.5, p=0.017$ ), comparable blood loss (322 vs. $308, p=0.545$ ), comparable blood transfusion ( $24 \%$ vs. $16 \%$,

Table 1 Sociodemographic and clinical parameters of patients

\begin{tabular}{|c|c|c|c|}
\hline $\begin{array}{l}\text { Sociodemographic and } \\
\text { clinical parameters }\end{array}$ & Pericranium & G-patch & $p$-Value \\
\hline \multicolumn{4}{|l|}{ Gender } \\
\hline Male & $22(88 \%)$ & $20(80 \%)$ & 0.702 \\
\hline Female & $3(12 \%)$ & $5(20 \%)$ & \\
\hline \multicolumn{4}{|l|}{ Age in years } \\
\hline $18-30$ & $8(32 \%)$ & $11(44 \%)$ & 0.484 \\
\hline $31-45$ & $8(32 \%)$ & $5(20 \%)$ & \\
\hline $46-60$ & $8(32 \%)$ & $6(24 \%)$ & \\
\hline $61-65$ & $1(4 \%)$ & $3(12 \%)$ & \\
\hline Headache & $15(60 \%)$ & $8(32 \%)$ & 0.089 \\
\hline Vomiting & $11(44 \%)$ & $13(52 \%)$ & 0.777 \\
\hline \multicolumn{4}{|l|}{ Category of head injury } \\
\hline Minimal head injury (15) & $11(44 \%)$ & $4(16 \%)$ & 0.036 \\
\hline $\begin{array}{l}\text { Mild head injury (14 Or } 15 \\
+ \text { Loc) }\end{array}$ & $3(12 \%)$ & $4(16 \%)$ & \\
\hline $\begin{array}{l}\text { Moderate head injury } \\
(9-13)\end{array}$ & $6(24 \%)$ & $15(60 \%)$ & \\
\hline Severe head injury (5-8) & $5(20 \%)$ & $2(8 \%)$ & \\
\hline
\end{tabular}


Table 2 Operative characteristics of patients

\begin{tabular}{|l|l|l|l|}
\hline Operative characteristics & Pericranium & G-patch & $p$-Value \\
\hline Duraplasty time in minutes (mean \pm SD) & $34.32 \pm 6.46$ & $27.80 \pm 6.42$ & 0.44 \\
\hline Drain output in mL (mean [min-max]) & $54.8(20-150)$ & $74.5(20-200)$ & 0.017 \\
\hline Re-exploration & $0(0 \%)$ & $0(0 \%)$ & - \\
\hline Blood loss (mean \pm SD) & $322 \pm 80.47$ & $308.33 \pm 76.14$ & 0.545 \\
\hline Blood transfusion & $6(24 \%)$ & $4(16 \%)$ & 0.48 \\
\hline Hospital stay (mean) & 8.6 & 10.44 & 0.028 \\
\hline
\end{tabular}

Table 3 Complications in patients

\begin{tabular}{|c|l|l|l|}
\hline Complications & Pericranium & G-patch & $p$-Value \\
\hline Wound infection & $2(8 \%)$ & $4(16 \%)$ & 0.384 \\
\hline CSF leak & $0(0 \%)$ & $2(8 \%)$ & 0.149 \\
\hline Seroma & $2(8 \%)$ & $4(16 \%)$ & 0.384 \\
\hline Seizure & $3(12 \%)$ & $2(8 \%)$ & 0.637 \\
\hline Wound gape & $2(8 \%)$ & $3(12 \%)$ & 0.637 \\
\hline
\end{tabular}

Abbreviations: CSF, cerebrospinal fluid.

$p=0.48)$, and significantly lesser duration of hospital stay ( 8.6 vs. $10.44, p=0.028$ ) (-Table 2 ).

Among the complications, compared with the patients undergoing G-patch, the patients who underwent pericranium autologous graft had comparable wound infection ( $8 \%$ vs. $16 \%, p=0.384$ ), comparable CSF leak ( $0 \%$ vs. $8 \%$, $p=0.149)$, comparable seroma ( $8 \%$ vs. $16 \%, p=0.384)$, comparable seizure ( $12 \%$ vs. $8 \%, p=0.637)$, and comparable wound gape ( $8 \%$ vs. $12 \%, p=0.637)$ (-Table 3 ).

\section{Discussion}

The relevance of increasing TBI was addressed in the International Consensus Meeting (2017), where guidelines pertaining to decompressive craniectomy (DC) were laid down to decrease the mortality and improve the outcomes by better managing the increased ICP after injury, perioperative care, and cranial reconstruction. ${ }^{1}$

After any such cranial procedure, an appropriate dural closure is important to decrease the risk of CSF leaks or fistulas and infections. Literature reports CSF leak among $4 \%$ in transsphenoidal procedures to $32 \%$ in posterior fossa procedures. ${ }^{7}$ It necessitates the need to improvise on the technique of dural closure and the use of dural substitutes with minimal complications. Among the various dural substitutes available, we compared the autologous and the synthetic grafts and found that galea-pericranium was better than bovine pericardium in terms of less hospital stay and less drain output volume among TBI patients. However, the complications were comparable among both the substitutes.

The use of different dural substitutes depends on the indications for duraplasty. In a recent article, it was determined that the most common indication for duraplasty was tumor resection (53\% cases) which was covered using synthetic grafts. ${ }^{8}$ Another included Arnold-Chiari malformations, which was managed commonly by allografts, and removal of subdural hematomas after TBI which were covered by xenografts. The pitfall in the review article was that the use of autografts was not assessed because of the evidence that pericranium has poor handling properties (being thin and fragile), harvesting it entails more time, material harvested is less to cover large gaps, and larger incidence of CSF fistulas, aseptic meningitis, and implant failures. It was stressed that certain advantages envisaged by the biological and synthetic grafts makes their use important, such as the incorporation of xenografts into native dura without the need for resuturing in trauma cases. We propose that the study may be biased due to the easy and high-availability of nonautologous grafts at their hospital which made them exclude the use of autologous grafts. Although time constraint is a significant factor to avoid the use of autologous grafts in a busy setup, it must be borne in mind that the use of allografts and xenografts carry a risk of spread of prion diseases. ${ }^{9}$ Thus, all the aspects need to be taken into account while choosing the type of dural graft in a developing country.

The choice of the dural substitute during a surgery must have a balance between the effectiveness and the safety features. The effectiveness of the dural substitutes may be judged by the surgery time, blood loss during the surgery, and the hospital stay. The index study found autologous graft to be better than synthetic graft in terms of less hospital stay, thus decreasing the overall cost of the procedure. While the safety of the dural substitutes depends on the incidence of complications such as wound infection, CSF leak and collection, wound gaping or infection, and occurrence of alternate events such as seizures. The current study found both types of substitutes to be similar with regard to all the complications. Our findings were in line with the study by Sabatino et al, who also found both autologous and nonautologous grafts to be comparable except for the high-cost of synthetic grafts. ${ }^{5}$ The average time to harvest in their study was just 2 minutes as compared with 5 minutes in the index study. 
There have been fewer recent research works that have compared the autologous and nonautologous grafts. However, some of the previous studies have suggested that the shortcomings of autologous grafts can be overcome and thus the use of autologous dural grafts is more appropriate. Zhang et al conducted a research about the extensive duraplasty with autologous grafts and found that with expertise and adequate time, larger areas of dural gap can be covered. ${ }^{10}$ Even Sabatino et al. showed that supratentorial approach can harvest autologous grafts large enough to cover any dural gaps. ${ }^{5}$ In another recent study, it was found that autologous graft (fascia lata) can be safe and effective for covering large dural defects which may be reinforced by fat grafts or fibrin sealants for effective closure, causing minimal CSF leaks and infections. ${ }^{11}$ Both the studies showed that the autologous grafts may be improvised to overcome the limitations without affecting the duraplasty outcomes. Kharal et al also stressed that the second incision for graft harvesting had no significant impact on the quality of life of the patients. ${ }^{11}$ Rather, such incisions have become common now due to the increasing elective hair transplant surgeries.

As far as the complication rates are concerned, the comparison of autologous and synthetic grafts shows increased infections and CSF leak with synthetic grafts. In a study by Malliti et al, the use of neuropatch was associated with increased deep wound infection (15\% vs. 5\%, $p=0.06$ ) and CSF leaks (13\% vs.1.6\%, $p<0.05) .{ }^{12}$ The reason was ascribed to the foreign material reaction to the implanted dural synthetic substitute. Considering this as an important factor, the ongoing research has focused on the development of different synthetic dural substitutes which may induce the least foreign body reaction and be the most effective.

With regard to the safety and effectiveness of the dural substitutes, it is always important to attain a watertight closure with good tensile strength. Factors such as diabetic status, infratentorial surgery, $>8$ days of postoperative corticosteroid, increased CRP, and the need for dural patch may increase the risk of CSF leak. This CSF leak increases the risk of development of an infection, causing morbidity and mortality. ${ }^{13}$ Thus, all the processes are interlinked; however, they are always measured separately.

The understanding of all such factors has led to better application and development of the artificial duraplasty substitutes in an effort to replace the use of autologous substitute. One of the studies found the equine pericardium to be an effective dural substitute with minimal wound infection and CSF leaks. ${ }^{6}$ One of the studies found that the use of biodegradable substances such as collagen matrix may subserve the purpose by providing a scaffold for the tissue regeneration without suturing. ${ }^{14}$ Collage matrix was found to be safe and effective dural substitute as none of the operated patients had CSF leak. Ammar studied fascia lata graft and fibrin glue as a substitute for dural repair in postoperative CSF leak after cranial surgery and found them to have minimal CSF leaks and infection. ${ }^{15}$ Kinaci et al, in a systematic review, found dural sealants to be effective in lowering the surgical site infections. ${ }^{16}$ Among the other materials, amniotic membrane and chitin has also been found provide an effective watertight covering with minimal CSF leak and infections. ${ }^{17,18}$ Such materials also help avoiding the need of resuturing. The latest report in 2019 said that Gore-Tex Expanded Cardiovascular Patch and Durepair, USA, have better biomechanical strength with similar thickness, impact absorbency and elasticity as human dura mater. ${ }^{4}$ Almost to the index study, the use of double layer G-patch has been reinforced in the one of the latest published studies ${ }^{19}$ where it was shown that double-layer G-patch during DC facilitates subsequent cranioplasty by preventing adhesions between the layers, resulting in easier dissection and reduced blood loss. The current and other study results support the importance of use of autologous grafts and the $\mathrm{G}$-patch in the current growing era of innovation of newer graft materials.

The current study holds strength in being prospective in nature and also because it shows the similar effectiveness and safety of the autologous grafts (vs. nonautologous grafts), but the results must be interpreted in view of certain limitations. First, the biomechanical properties of the dural grafts were not taken into account. Second, comparison among large and small dural gaps was not done. Third, follow-ups of the patients were for a small period of time.

\section{Conclusion}

In conclusion, the study showed that galea-pericranium and polypropylene dural patch are equally effective and safe dural substitutes in providing a dural seal to minimize the CSF leaks and infections among posttraumatic brain injury patients.

\section{Funding \\ None. \\ Conflict of Interest \\ None declared.}

\section{References}

1 Hutchinson PJ, Kolias AG, Tajsic T, et al. Consensus statement from the International Consensus Meeting on the Role of Decompressive Craniectomy in the Management of Traumatic Brain Injury: Consensus statement. Acta Neurochir (Wien) 2019;161(7):1261-1274

2 Sinha S, Raheja A, Garg M, et al. Decompressive craniectomy in traumatic brain injury: A single-center, multivariate analysis of 1,236 patients at a tertiary care hospital in India. Neurol India 2015;63(2):175-183

3 Sun $\mathrm{H}$, Wang $\mathrm{H}$, Diao $\mathrm{Y}$, et al. Large retrospective study of artificial dura substitute in patients with traumatic brain injury undergo decompressive craniectomy. Brain Behav 2018;8(5):e00907

4 Kizmazoglu C, Aydin HE, Kaya I, et al. Comparison of biomechanical properties of dura mater substitutes and cranial human dura mater: an in vitro study. J Korean Neurosurg Soc 2019;62(6):635-642

5 Sabatino G, Della Pepa GM, Bianchi F, et al. Autologous dural substitutes: a prospective study. Clin Neurol Neurosurg 2014;116:20-23

6 Centonze R, Agostini E, Massaccesi S, Toninelli S, Morabito L. A novel equine-derived pericardium membrane for dural 
repair: a preliminary, short-term investigation. Asian J Neurosurg 2016;11(3):201-205

7 Ha B-J, Cheong JH, Yi H-J. Risk factors for cerebrospinal fluid leakage after craniotomy and the efficacy of dural sealants application versus dural suturing alone. Nerve 2016;2:22-25

8 Azzam D, Romiyo P, Nguyen T, et al. Dural repair in cranial surgery is associated with moderate rates of complications with both autologous and nonautologous dural substitutes. World Neurosurg 2018;113:244-248

9 Bonda DJ, Manjila S, Mehndiratta P, et al. Human prion diseases: surgical lessons learned from iatrogenic prion transmission. Neurosurg Focus 2016;41(1):E10

10 Zhang GL, Yang WZ, Jiang YW, Zeng T. Extensive duraplasty with autologous graft in decompressive craniectomy and subsequent early cranioplasty for severe head trauma. Chin J Traumatol 2010;13(5):259-264

11 Kharal IA, Bhatti A, Saeed S, et al. Use of large fascia lata graft as dural substitute in neurosurgical procedures at Neurosurgery Department Teaching Hospital D G Khan. Pakistan J Neurological Sci 2017;21(2)

12 Malliti M, Page P, Gury C, Chomette E, Nataf F, Roux FX. Comparison of deep wound infection rates using a synthetic dural substitute (neuro-patch) or pericranium graft for dural closure: a clinical review of 1 year. Neurosurgery 2004;54(3): 599-603, discussion 603-604
13 Bangash M, Alkhotani A. Risk factors affecting the postoperative cerebrospinal fluid leak in brain surgery. Int J Contemp Med Res 2016;3(5):1522-1525

14 Stendel R, Danne M, Fiss I, et al. Efficacy and safety of a collagen matrix for cranial and spinal dural reconstruction using different fixation techniques. J Neurosurg 2008;109(2):215-221

15 Ammar AS. Fibrin glue and fascia lata graft for management of cerebrospinal fluid leakage after cranial surgery, preliminary results. Menoufia Med J 2016;29:637-641

16 Kinaci A, Algra A, Heuts S, O’Donnell D, van der Zwan A, van Doormaal T. Effectiveness of dural sealants in prevention of cerebrospinal fluid leakage after craniotomy: a systematic review. World Neurosurg 2018;118:368-376.e1

17 Turchan A, Rochman TF, Ibrahim A, et al. Duraplasty using amniotic membrane versus temporal muscle fascia: a clinical comparative study. J Clin Neurosci 2018;50:272-276

18 Pogorielov M, Kravtsova A, Reilly GC, et al. Experimental evaluation of new chitin-chitosan graft for duraplasty. J Mater Sci Mater Med 2017;28(2):34

19 Pathrose Kamalabai R, Nagar M, Chandran R, et al. Rationale behind the use of double-layer polypropylene patch (G-patch) dural substitute during decompressive craniectomy as an adhesion preventive material for subsequent cranioplasty with special reference to flap elevation time. World Neurosurg 2018;111 :e105-e112 